\title{
Dynamic formation of liquid droplets triggered by sequential enzymatic reactions
}

\author{
Tomoto Ura, ${ }^{\dagger,}$ Shunsuke Tomita, ${ }^{\S}$ and Kentaro Shiraki ${ }^{\dagger}$
}

${ }^{\dagger}$ Faculty of Pure and Applied Sciences, University of Tsukuba, 1-1-1 Tennodai, Tsukuba, Ibaraki 305-8573, Japan.

$\S$ Biomedical Research Institute, National Institute of Advanced Industrial Science and Technology, 1-1-1 Higashi, Tsukuba, Ibaraki 305-8566, Japan.

Corresponding Author. Email: shiraki@bk.tsukuba.ac.jp

\begin{abstract}
A model system was developed that dynamically generates two different liquid droplets via liquid-liquid phase separation coupled with a sequential glycolytic reaction. The sequential two-enzyme reaction triggers the formation/dissolution of the liquid droplets. The droplets, in turn, compartmentalize each enzymatic step and generate feedback to accelerate the overall reaction.
\end{abstract}


Biological reactions are tightly regulated in all organisms. ${ }^{1}$ The interior of cells is a crowded environment with many biomolecules at high concentrations. ${ }^{2}$ At present, there is no clear explanation why hundreds or thousands of enzymatic reactions proceed without unexpected crosstalk in cells. One plausible hypothesis is that enzymes are compartmentalized in specific locations within the cells. In other words, enzymes may assemble as needed to produce the sequential reactions. ${ }^{3}$ Such assembly would be expected to enhance reaction efficiency by keeping groups of molecules involved in enzymatic reactions, including reaction intermediates, in proximity. ${ }^{4-6}$ Recent advances in bioimaging have enabled observation of such "metabolon" and quaternary structures in vivo; however their assembly details remain ambiguous. ${ }^{3}$

Liquid-liquid phase separation (LLPS) provides new insights into biological compartmentalization in cells. $^{7}$ LLPS is a thermodynamic phenomenon in which molecules congregate to form a liquid phase separated from the bulk phase. ${ }^{8}$ Many kinds of liquid droplets enriched in proteins and nucleic acids are now known to form and disperse reversibly in cells in response to environmental changes. These droplets are involved in various biological processes, such as gene expression ${ }^{9,10}$, signal transduction ${ }^{11}$, and immune responses ${ }^{12}$. To understand how these LLPS are coupled with biochemical reactions, their reconstruction in vitro is essential.

Enzymatic reactions and droplet generations can interact inside cells. The progress of enzymatic reactions controls the formation or dissolution of droplets, while the incorporation of enzymes into liquid droplets promotes or inhibits reactions. As examples, a protease dispersed droplets by hydrolysis of proteins constituting the droplets ${ }^{13,14}$, and phosphorylation and dephosphorylation of droplet components by kinases and phosphatases, resulting from the stepwise addition of necessary factors, regulated the formation and dissolution of liquid droplets. ${ }^{15,16}$ Further, liquid droplets can affect enzymatic reactions, as observed in kinase,${ }^{17}$ ribozymes,${ }^{18}$ multi-enzyme complexes, ${ }^{19}$ and sequential reactions ${ }^{20,21}$ In these reports, liquid droplets acted as reaction fields for enzymes, separate from the bulk solution. However, much of the studies has focused primarily on the unidirectional action of enzymes or droplets, and there is not yet sufficient knowledge regarding the dynamic relationship between changes in sequential enzymatic activity due to incorporation into liquid droplets and changes in the states of the droplets due to 
enzymatic conversion of droplet components. These relationships are important because droplet formation/dissolution via LLPS is expected for many sequential enzymatic reactions.

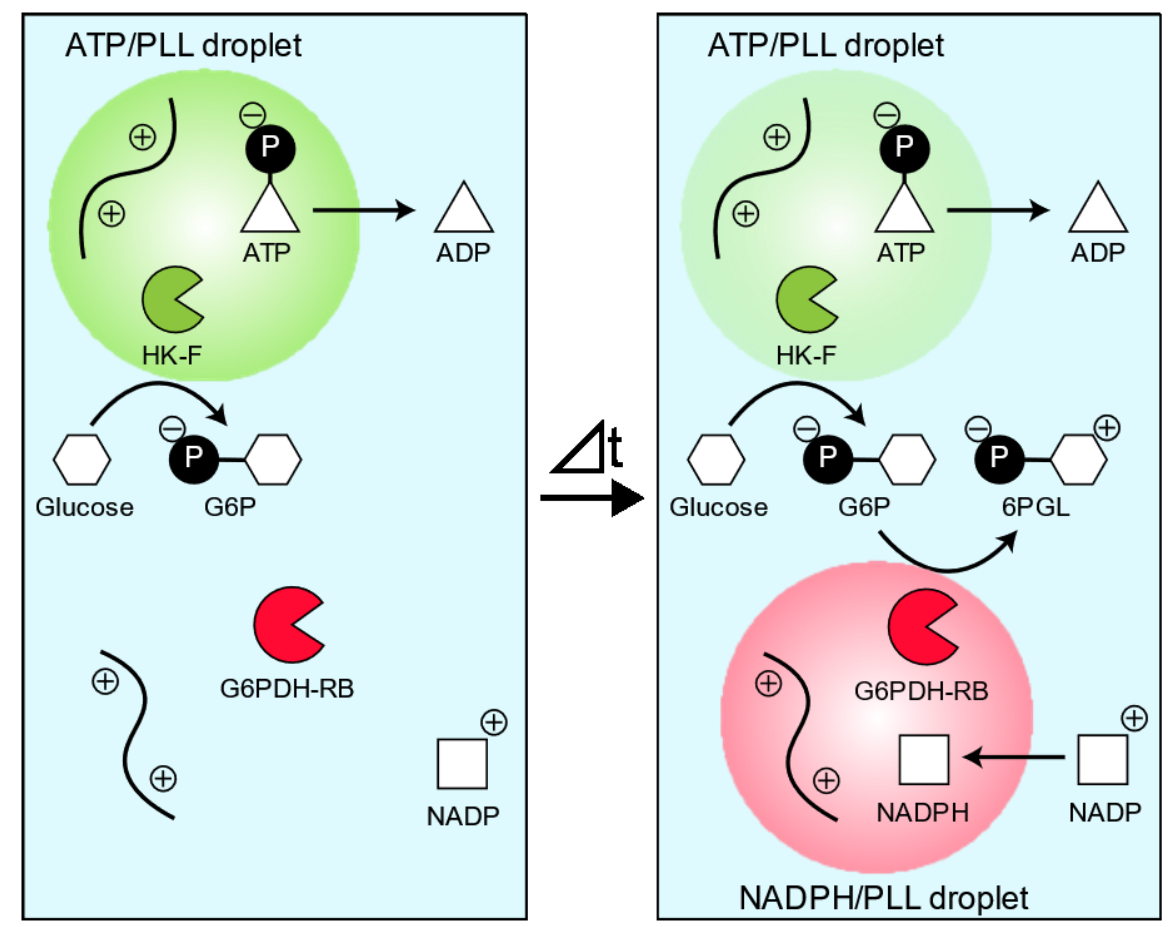

Figure 1. Schematic of the dynamic formation/dissolution of different liquid droplets associated with the sequential glycolytic reaction. Initially, the phosphate transfer reaction (from ATP to ADP) catalyzed by FITC-modified HK (HK-F) proceeds with the dissolution of ATP/PLL droplets. The increase in intermediate G6P leads to the electron transfer reaction (from NADP to NADPH) catalyzed by RBITC-modified (G6PDH-RB) to form NADPH/PLL droplets.

In this study, a model system was constructed where liquid droplets containing enzymes form and disperse via LLPS in response to enzymatic reactions, and the formation/dissolution of the liquid droplets feeds back on enzymatic reaction rate (Figure 1). Liquid droplets were produced using anionic nucleotides (ATP, ADP, NADP, NADPH, NAD, and NADH) and cationic poly-L-lysine (PLL) (for the chemical structures, see Fig S1, ESI). These nucleotides are abundant in living cells; ATP and ADP serve as energy currency, while NADP, NADPH, NAD, and NADH are critical for electron transport. Liquid droplets formed between ATP/ADP and PLL are a known in vitro model system, ${ }^{16,17,19,22}$ but droplets generated by NADP/NADPH have not been reported previously. A sequential enzymatic reaction of 
sugars and the nucleotides by hexokinase (HK) and glucose-6 phosphate dehydrogenase (G6PDH) in the presence of nucleotide/PLL droplets was investigated, where the nucleotides serve as the substrates for the enzymes as well as the factors required for droplet formation. The formation/dissolution of droplets occurred dynamically during the enzymatic reaction, and sequential glycolysis by HK and G6PDH was accelerated in the presence of droplets (Figure 1).

LLPS of nucleotides and PLL was initially examined by optical microscopy (Figure 2A), with the samples contained $0.5 \mathrm{mM}$ of the various nucleotides with concentrations of PLL ranging from 0 to 5 $\mathrm{mM}$ (concentration refers to the monomer unit) in HEPES buffer at $\mathrm{pH}$ 7.0. ATP formed more droplets than ADP; droplets were observed in the range of 4-8 equiv of $-\mathrm{NH}_{3}{ }^{+}$for ATP, and 4-6 equiv for ADP. Consistent with previous studies, ${ }^{16,17}$ the greater the number of anionic phosphate groups, the greater the ability to form droplets with PLL. This trend was similar for NADPH/NADH; NADPH formed droplets over a very wide range (4-10 equiv), but NADH did not. Interestingly, when NADPH was oxidized to NADP, no droplets were observed except in the case of 4 equiv. The increase in positive charge due to oxidation attenuated the interaction with PLL (Figures 1 and S1, ESI). Consistent with this result, NAD did not form droplets.

The loss of nucleotides in the supernatant after centrifugation was consistent with microscopic observations (Figure 2B). Precipitation rates of ATP, ADP, and NADPH similarly increased with increasing PLL concentration, and maximum precipitation rates were NADPH $>$ ATP $>$ ADP. NADP, NAD, and NADH hardly precipitated. Based on these results, concentrations of 5 equiv of PLL against 
substrates (0.5 mM ATP and 0.5 mM NADP) were further examined for their effects on enzymatic reactions.

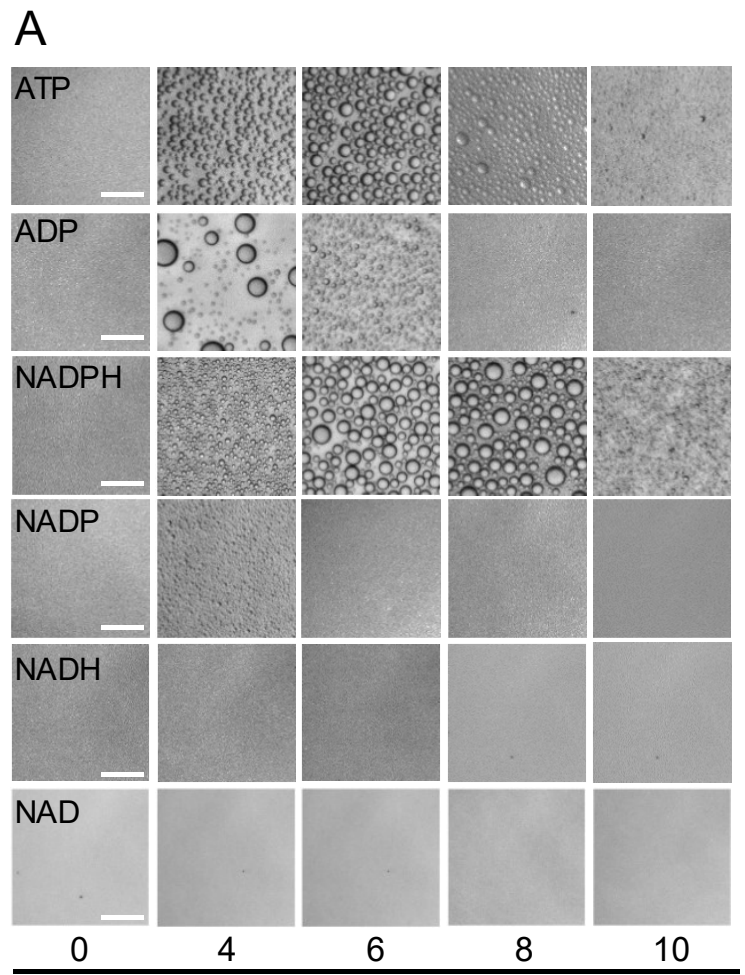

PLL/Nucleotide
B

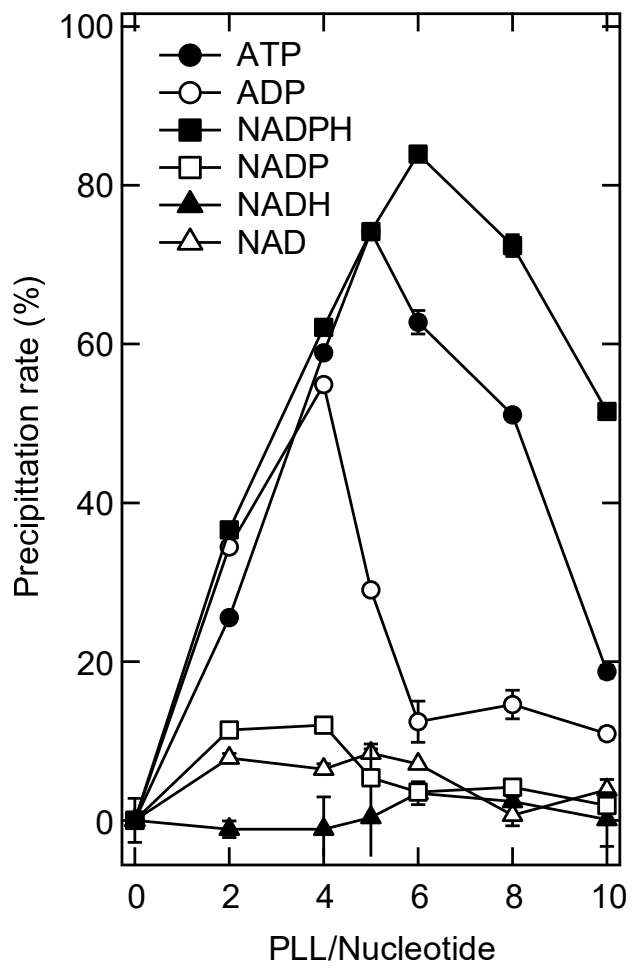

Figure 2. Formation of nucleotide/PLL droplets. (A) Optical microscopy images of nucleotide-PLL droplets. Scale bar, $10 \mu \mathrm{m}$ (B) Precipitation rate (\%) of nucleotides in the presence of PLL. All solutions were prepared with $0.5 \mathrm{mM}$ nucleotides, PLL concentrations between 0-5 mM (monomer), and $10 \mathrm{mM}$ HEPES (pH 7.0).

The formation of droplets and the localization of enzymes were observed during each enzymatic reaction. HK catalyzes a phosphorylation reaction that converts ATP and D-glucose to ADP and D-glucose-6phosphate (G6P) (Figure 3A). Fluorescein isothiocyanate (FITC) was partially introduced into HK (HKF) to track its localization. HK-F was mixed with PLL, ATP, D-glucose, and monitored by bright fieldand fluorescence microscopy (Figure 3B). Significantly, prior to the addition of D-glucose ( $\mathrm{t}=0 \mathrm{~min})$, HK-F was concentrated inside the PLL/ATP droplets. According to a line profile of fluorescence intensities (Figure 3C), fluorescence inside the droplets was markedly higher than outside. Upon the addition of D-glucose, droplets gradually dispersed, and HK-F was slowly released into the bulk phase 
(Figure 3B). At $t=10 \mathrm{~min}$, droplets almost completely disappeared and green fluorescence covered the entire field of view. The marked increase in fluorescence intensity after dispersion of the droplets was probably due to self-quenching of FITC inside the condensed droplets.

G6PDH catalyzes a redox reaction that converts NADP and G6P to NADPH and 6-phospho-Dglucono-1,5-lactone (6PGL) (Figure 3D). Here rhodamine B isothiocyanate (RBITC) was partially modified in G6PDH (G6PDH-RB) to distinguish it from HK-F. When PLL, NADP and G6PDH-RB were mixed ( $\mathrm{t}=0 \mathrm{~min}$ ), very small droplets with localized G6PDH-RB were observed (Figure 3E). In contrast to the behavior of HK-F, gradual generation and growth of G6PDH-RB-localized droplets were observed after G6P was added. At $\mathrm{t}=10 \mathrm{~min}$, the fluorescence intensity inside the generated droplets was also significantly higher than outside. Droplet formation was not observed for NAD which has less negative charge (Fig.S2, ESI).

A

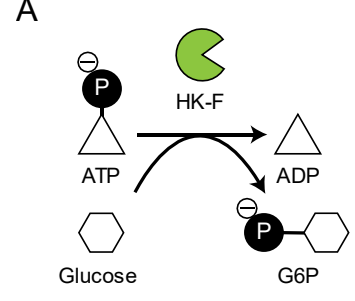

B

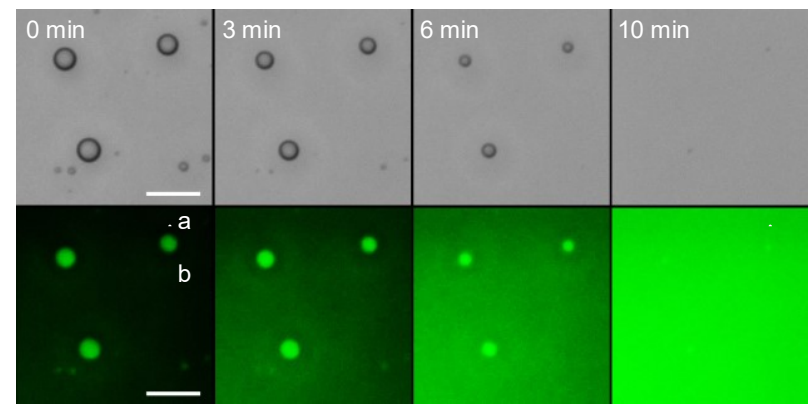

$\mathrm{C}$

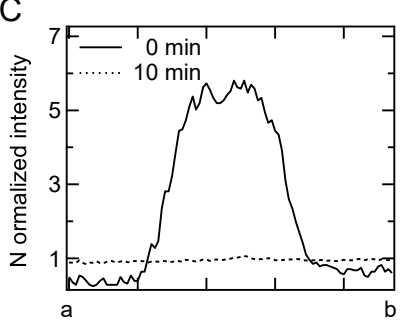

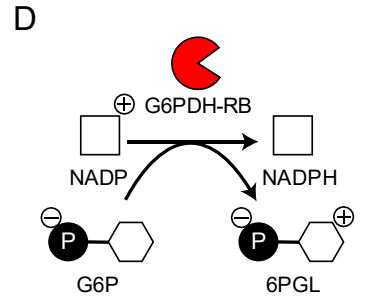

E
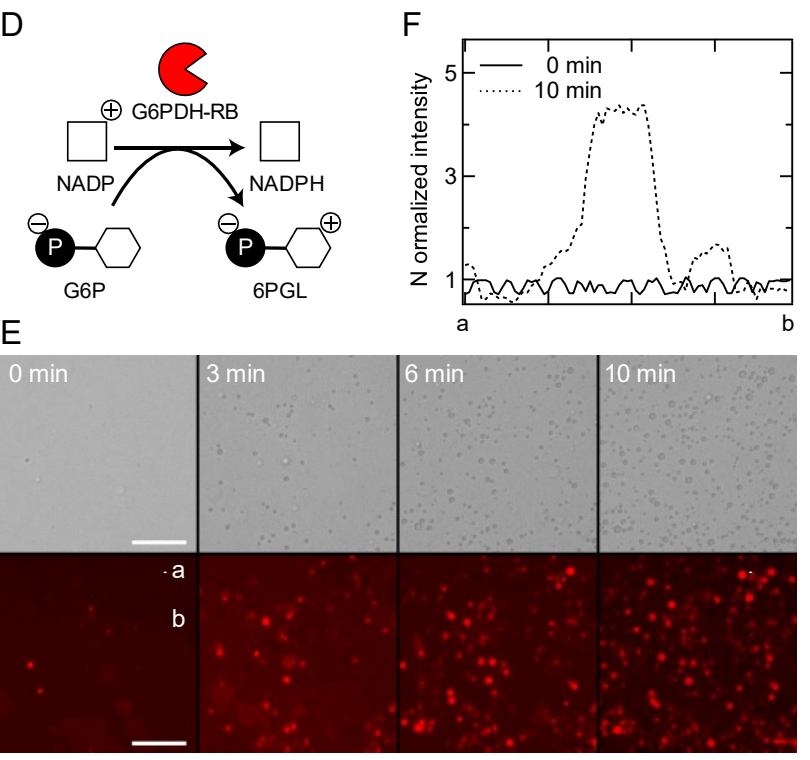

Fig. 3 The formation and dissolution of nucleotide/PLL droplets as the enzymatic reaction proceeds. Enzymatic reactions by HK (A) and G6PDH (D). Microscopic observation of droplet changes accompanied by the enzymatic reactions of HKF (B) and G6PDH-RB (E). Scale bar is $20 \mu \mathrm{m}$. Localization of HK-F (C) and G6PDH-RB (F) in droplets. Fluorescence intensity before $(0 \mathrm{~min})$ and after reaction $(10 \mathrm{~min})$ along the broken white lines was quantified from the brightness of each pixel. 
The reverse effect of HK-F and G6PDH-RB on LLPS lead to the reproduction of droplet dissolution/generation coupled with these enzymes and investigation of the effect of the sequential reaction on droplets, and associated reaction efficiency. During the sequential conversion of D-glucose to 6PGL, ATP is dephosphorylated to ADP and NADP (or NAD) is reduced to NADPH (or NADH) (Figure 4A). In the presence of PLL, ATP, NADP, HK-F, and G6PDH-RB (i.e., no D-glucose), large droplets containing HK-F and small droplets containing G6PDH-RB were observed $(\mathrm{t}=0$ min, Figures 4B and S3, ESI), suggesting that PLL/ATP droplets and PLL/NADP droplets are selective for HK-F and G6PDH$\mathrm{RB}$, respectively. This unexpected selectivity might be due to specific binding of enzymes to substrates. As reactions proceeded, some droplets became smaller, and, at the same time, new droplets formed and grew (Figures 4B and S4, for clarity, see movies S1-S3, ESI). Fluorescence images showed that reduced droplets contained HK-F and background green fluorescence gradually increased. Moreover, newly formed droplets contained G6PDH-RB. Thus, dissolution and formation of droplets with different properties occurred dynamically and were coupled to sequential enzymatic reaction. Surprisingly, HK-F and G6PDH-RB were clearly compartmentalized, even though droplets were both PLL-based. Such compartmentalization seems to be similar to what is expected in cells. The incomplete disappearance of HK-F-containing droplets may be due to changes in their internal compositions such as the incorporation of NADPH that is the final product of the sequential reaction. This hypothesis is supported by the results of using NAD instead of NADP (Figure S5 ESI), where HK-F-containing droplets disappeared and G6PDH-RB-containing droplets did not generate. 

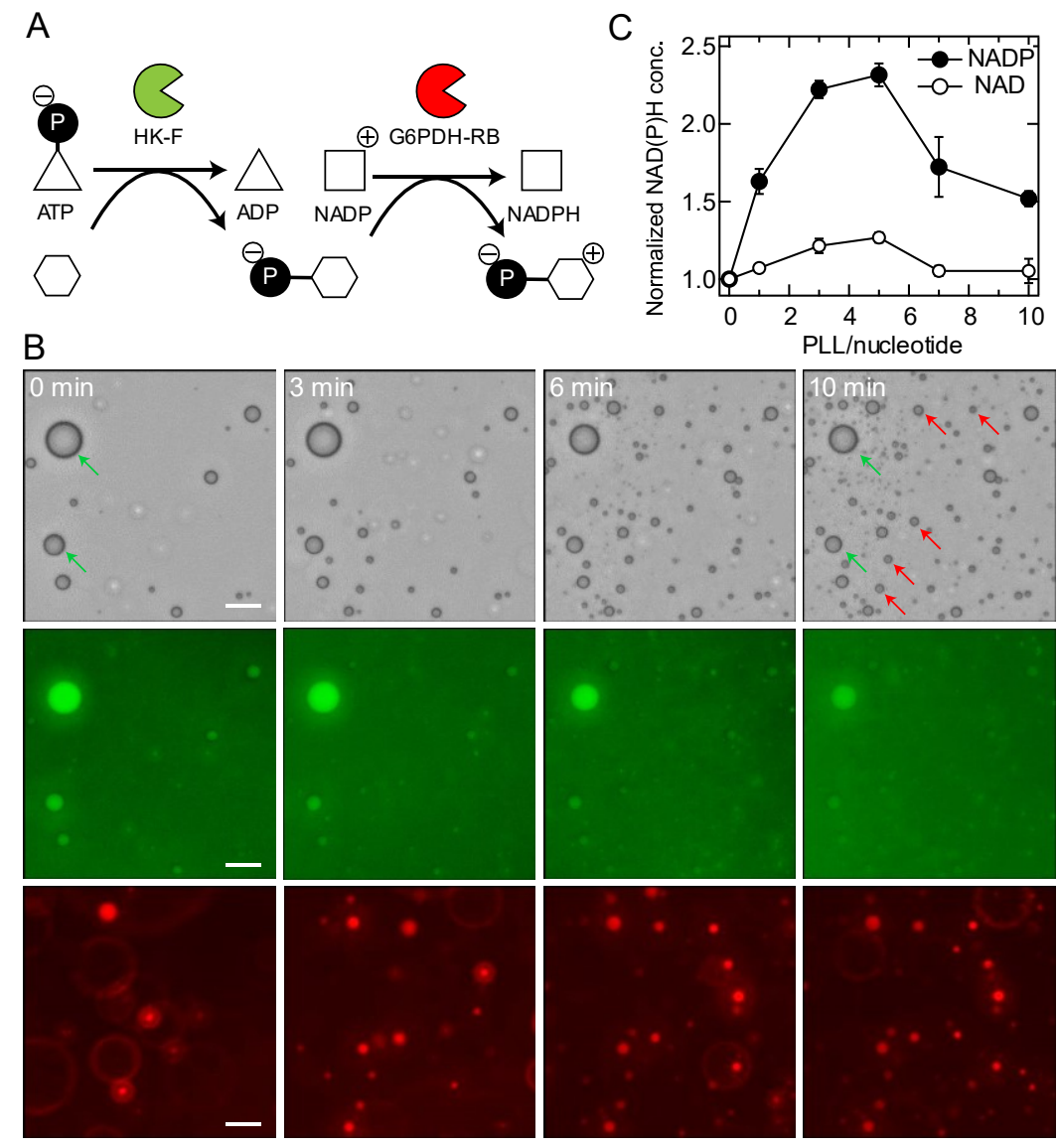

Fig. 4 Coupling of sequential reaction and nucleotide/PLL droplet generation. (A) Scheme of a sequential reaction by HK and G6PDH. (B) Changes in nucleotide-PLL droplets and enzyme localization with the progress of the sequential reaction. Scale bar is $20 \mu \mathrm{m}$. The green and red arrows point to the HK-F-containing droplets and the newly generated G6PDH-RB containing droplets, respectively. (C) Normalized concentration of the final product of the sequential reaction in the presence of PLL.

Finally, how the concentration of LLPS affects the production of NADPH (or NADH) (Figure 4C and S6, ESI) was explored. When NADP was used as the substrate, the maximum amount of NADPH increased 2.2-folds for the 5 equiv PLL (same conditions in Fig. 4B), compared with the case without PLL. However, the amount of NADH increased only 1.2-fold when using NAD that did not form droplets. The formation of droplets that were selective to each enzyme/substrate pair, which was coupled to the progression of the sequential enzymatic reaction, apparently, improved catalytic efficiency by compartmentalizing each enzymatic reaction system. 
When electrostatically complementary charged polymers and substrates coexist, enzyme activity is increased by a decrease in the Michaelis constant. ${ }^{23}$ Anionic substrates (ATP, NADP, and G6P) and cationic PLL used in this study are similar materials used previously. Further, the compartmentalization of enzyme systems into different phases may prevent substrate ${ }^{21}$ or possibly product inhibition. Indeed, enzyme activities of G6PDH and HK decrease with increasing concentration of $\mathrm{ATP}^{24}$ and $\mathrm{ADP}^{25}$, respectively. Ongoing research is exploring the detailed impact of droplet compartmentalization on each enzymatic process to better understand the effects of the above factors.

In summary, we have developed a system in which a sequential glycolytic reaction catalyzed by two enzymes (HK and G6PDH) induces the dissolution/formation of droplets. These droplets can compartmentalize each reaction and allow feed back between reactions. The enzyme system coupled with LLPS may be a key to unraveling sequential biochemical reactions in living cells that contain numerous molecules. In addition, it may also support development of important industrial applications, such as energy production, ${ }^{26}$ chemical manufacturing, ${ }^{27}$ synthetic biology, ${ }^{28}$ and metabolic engineering. ${ }^{29}$ For G6PDH, an oxidoreductase used to extract bioenergy from biomolecules, ${ }^{30}$ it may be possible to improve production efficiency of simply by adding appropriate charged polymers without relying on introducing mutations. ${ }^{31}$ Overall, our strategy for constructing LLPS-coupled enzyme sequential reactions offers new perspectives on enzymatic reactions in living cells and may offer a means to increase the efficiency of industrial enzyme use. The study was supported JSPS KAKENHI (grant number 18H02383 and 19K22377). 
References

1 J. Ovádi and V. Saks, Mol. Cell. Biochem., 2004, 256/257, 5-12.

2 D. S. Goodsell, Trends Biochem. Sci., 1991, 16, 203-206.

3 D. L. Schmitt and S. An, Biochemistry, 2017, 56, 3184-3196.

4 M. Castellana, M. Z. Wilson, Y. Xu, P. Joshi, I. M. Cristea, J. D. Rabinowitz, Z. Gitai and N. S. Wingreen, Nat. Biotechnol., 2014, 32, 1011-1018.

5 F. Hinzpeter, U. Gerland and F. Tostevin, Biophys. J., 2017, 112, 767-779.

6 L. Poshyvailo, E. Von Lieres and S. Kondrat, PLoS One, 2017, 12, 1-17.

7 Y. Shin and C. P. Brangwynne, Science (80-. )., 2017, 357, eaaf4382.

8 S. F. Banani, H. O. Lee, A. A. Hyman and M. K. Rosen, Nat. Rev. Mol. Cell Biol., 2017, 18, 285298.

9 C. J. Decker and R. Parker, Cold Spring Harb. Perspect. Biol., 2012, 4, a012286.

10 D. Hnisz, K. Shrinivas, R. A. Young, A. K. Chakraborty and P. A. Sharp, Cell, 2017, 169, $13-23$.

11 X. Su, J. A. Ditlev, E. Hui, W. Xing, S. Banjade, J. Okrut, D. S. King, J. Taunton, M. K. Rosen and R. D. Vale, Science, 2016, 352, 595-599.

12 M. Du and Z. J. Chen, Science., 2018, 361, 704-709.

13 S. N. Semenov, A. S. Y. Wong, R. M. Van Der Made, S. G. J. Postma, J. Groen, H. W. H. Van Roekel, T. F. A. De Greef and W. T. S. Huck, Nat. Chem., 2015, 7, 160-165. 
14 B. S. Schuster, E. H. Reed, R. Parthasarathy, C. N. Jahnke, R. M. Caldwell, J. G. Bermudez, H. Ramage, M. C. Good and D. A. Hammer, Nat. Commun., 2018, 9, 2985.

15 W. M. Aumiller and C. D. Keating, Nat. Chem., 2016, 8, 129-137.

16 K. K. Nakashima, J. F. Baaij and E. Spruijt, Soft Matter, 2018, 14, 361-367.

17 S. Koga, D. S. Williams, A. W. Perriman and S. Mann, Nat. Chem., 2011, 3, 720-724.

18 C. A. Strulson, R. C. Molden, C. D. Keating and P. C. Bevilacqua, Nat. Chem., 2012, 4, 941-946.

19 J. Crosby, T. Treadwell, M. Hammerton, K. Vasilakis, M. P. Crump, D. S. Williams and S. Mann, Chem. Commun., 2012, 48, 11832-11834.

20 W. M. Aumiller, B. W. Davis, N. Hashemian, C. Maranas, A. Armaou and C. D. Keating, J. Phys. Chem. B, 2014, 118, 2506-2517.

21 T. Kojima and S. Takayama, ACS Appl. Mater. Interfaces, 2018, 10, 32782-32791.

22 E. A. Frankel, P. C. Bevilacqua and C. D. Keating, Langmuir, 2016, 32, 2041-2049.

23 T. Kurinomaru, S. Tomita, Y. Hagihara and K. Shiraki, Langmuir, 2014, 30, 3826-3831.

24 A. Gad, Proc. Natl. Acad. Sci. U. S. A., 1966, 56, 1543-1547.

25 H. J. FROMM and V. ZEWE, J. Biol. Chem., 1962, 237, 3027-3032.

26 D. Sokic-Lazic and S. D. Minteer, Biosens. Bioelectron., 2008, 24, 939-944.

27 F. López-Gallego, E. Jackson and L. Betancor, Chem. - A Eur. J., 2017, 23, 17841-17849. 
28 C. M. Agapakis, P. M. Boyle and P. A. Silver, Nat. Chem. Biol., 2012, 8, 527-535.

29 H. Lee, W. C. DeLoache and J. E. Dueber, Metab. Eng., 2012, 14, 242-251.

30 Jiafu Shi, Yizhou Wu, Shaohua Zhang, Yu Tian, Dong Yang and Zhongyi Jiang, Chem. Soc. Rev., $2018,47,4295-4313$.

31 Z. Zhu and Y. H. P. Zhang, Metab. Eng., 2017, 39, 110-116. 
TOC

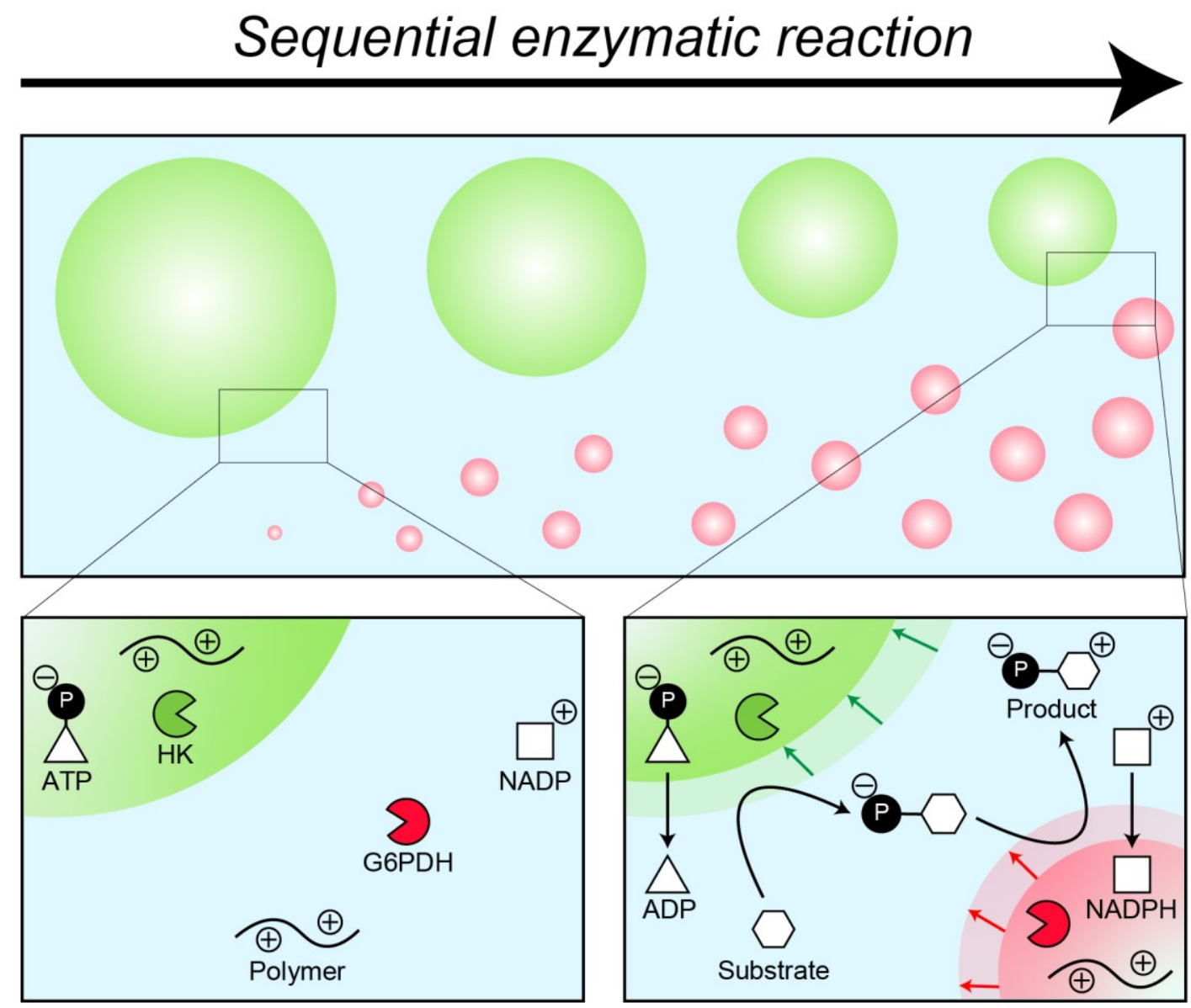

Schematic of dynamic formation/dissolution of different liquid droplets triggered by the sequential glycolytic reaction. ATPbased droplets gradually dissolve, while NADPH-based droplets generate as the sequential reaction proceeds, which accelerates the production of final product, NADPH. 